complete vascular ring. The patients can have tracheal or bronchial malacia or deformed cartilage rings with tracheal stenosis leading to incomplete resolution of presenting symptoms with standard surgical techniques. From a diagnostic standpoint, rigid intraoperative bronchoscopy was critical to identify these residual lesions and allowed patient-specific surgical management. The stated goal was to produce an airway where more than $50 \%$ of the luminal diameter is free of extrinsic compression. In their report, the techniques used to accomplish this included the aortic uncrossing procedure, anterior or posterior tracheobronchopexy, or slide tracheoplasty. It is important to emphasize that these additional procedures are adjuncts to the standard surgical approach for each type of vascular anomaly. For example, in patients with a double aortic arch variant of a circumflex aorta, the aortic uncrossing procedure was undertaken after division of the nondominant arch and ligamentum arteriosum. Patients who continued to demonstrate significant airway compromise had additional surgical procedures based on the anatomy of the patient's airway. The 3-phase bronchoscopy advocated by the authors should be a central component of how congenital heart surgeons manage patients with a circumflex aorta or double aortic arch who may not be as straightforward as once thought.

\section{References}

1. Backer CL, Russell HM, Wurlitzer KC, Rastatter JC, Rigsby CK. Primary resection of Kommerell diverticulum and left subclavian artery transfer. Ann Thorac Surg. 2012;94:1612-7.

2. Callahan CP, Merritt TC, Canter MW, Eghtesady P, Manning PB, Abarbanell AM Symptom persistence after vascular ring repair in children. J Pediatr Surg. January 10, 2020 [Epub ahead of print].

3. Binsalamah ZM, Ibarra C, John R, Zea-Vera R, Adachi I, Imamura M, et al Contemporary midterm outcomes in pediatric patients undergoing vascular ring repair. Ann Thorac Surg. 2020;109:566-72.

4. Kamran A, Friedman KG, Jennings RW, Baird CW. Aortic uncrossing and tracheobronchopexy corrects tracheal compression and tracheobronchomalacia associated with circumflex aortic arch. J Thorac Cardiovasc Surg. 2020;160: 796-804.

\title{
Commentary: A long haul and a short slide?
}

\section{Michael E. Mitchell, MD}

In "Aortic Uncrossing and Tracheobronchopexy Corrects Tracheal Compression and Tracheobronchomalacia Associated with Circumflex Aortic Arch," Kamran and colleagues $^{1}$ make a strong case for a significant upgrade in the intensity of surgery versus a traditional approach for a subgroup of patients requiring surgical relief of severe airway compression due to a circumflex aortic arch. Their argument is carefully constructed, and the

\footnotetext{
From the Department of Surgery, Medical College of Wisconsin, and Division of Pediatric Cardiothoracic Surgery, Herma Heart Institute, Children's Hospital of Wisconsin, Milwaukee, Wis.

Disclosures: The author reported no conflicts of interest.

The Journal policy requires editors and reviewers to disclose conflicts of interest and to decline handling or reviewing manuscripts for which they may have a conflict of interest. The editors and reviewers of this article have no conflicts of interest.

Received for publication May 7, 2020; revisions received May 7, 2020; accepted for publication May 8, 2020; available ahead of print May 15, 2020.

Address for reprints: Michael E. Mitchell, MD, Department of Surgery, Medical College of Wisconsin, and Division of Pediatric Cardiothoracic Surgery, Herma Heart Institute, Children's Hospital of Wisconsin, 9000 W Wisconsin Ave, MS 715, Milwaukee, WI 53226 (E-mail: mmitchell@chw.org).

J Thorac Cardiovasc Surg 2020;160:807-8

$0022-5223 / \$ 36.00$

Copyright (c) 2020 by The American Association for Thoracic Surgery

https://doi.org/10.1016/j.jtcvs.2020.05.006
}

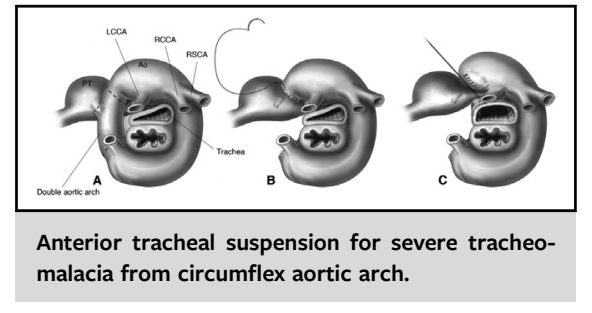

CENTRAL MESSAGE

Aortic uncrossing, combined with advanced tracheal procedures, might play an important role when performed correctly and applied selectively.

anatomic drawings and honesty of their presentation are excellent.

The authors identify that patients with severe respiratory symptoms and circumflex aortic arch are the patients in whom traditional repair of the vascular ring will tend to fail because of residual airway compression. ${ }^{1}$ They 

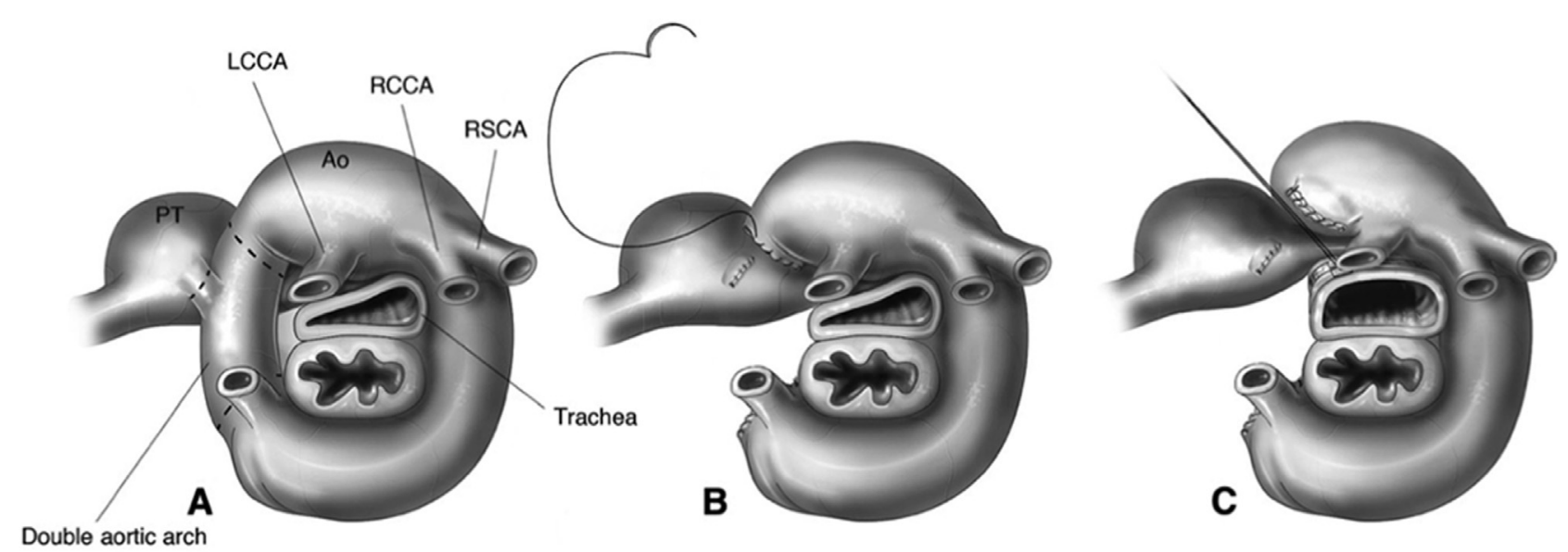

FIGURE 1. Anterior tracheal suspension for severe tracheomalacia from circumflex aortic arch. A, Right dominant double aortic arch with circumflex aorta. B, Division of double aortic arch. C, Anterior tracheal suspension. PT, Pulmonary trunk; LCCA, left common carotid artery; Ao, aorta; RCCA, right common carotid artery; RSCA, right subclavian artery.

note that the case for aortic uncrossing was first reported by Planche and LaCoeur-Gayet ${ }^{2}$ in 1984. Backer and colleagues $^{3}$ also reported that 2 of the 26 reinterventions in a series of 300 patients undergoing repair of the vascular ring had had a circumflex aorta. They reported their experience with 5 patients in whom the aortic uncrossing procedure was performed, 1 of whom had required aortic arch revision and 1 who had required a temporary tracheostomy.

It is logical that the traditional division of the double arch would not result in the same anatomic and physiologic relief experienced by patients with aortas descending on the same side; however, other less-invasive options are available. For example, anterior tracheal suspension, combined with standard ring repair, in this group of patients has had some success (Figure 1)., If aortic uncrossing could be performed with low morbidity, it would likely have been more broadly adopted, in particular, for the most severe cases. In the current series, 3 of 8 patients $(38 \%)$ had experienced major events, including $2(25 \%)$ who developed permanent bilateral vocal cord paralysis requiring tracheostomy. ${ }^{1}$ The authors have improved their technique, and these complications occurred early in the series $^{1}$; however, this learning curve will have to be overcome by others as well. To this point, the authors acknowledge that they do not recommend aortic uncrossing for all patients. ${ }^{1}$

We are left with the observation that the traditional division of the double aortic arch has resulted in the need for reintervention in some of these patients but we do not know what proportion. Thus, the question remains: in patients with a circumflex aorta, what is the percentage who have undergone traditional repair with division of a vascular ring, who have subsequently required reintervention after that repair?

The authors have added tracheopexy, both anterior and posterior, to the repair, as well as slide tracheoplasty when needed. ${ }^{1}$ These are essential innovations, although it could be the case that these advanced tracheal procedures could provide successful results in some patients without the need for aortic uncrossing.

This contribution is important both in describing a successful technique and experience in a very challenging group of patients and in openly discussing the improvements made to the approach over time, which have resulted in improved outcomes. The authors are to be applauded. It is a technique that will play a very important role when performed correctly and applied selectively.

\section{References}

1. Kamran A, Friedman K, Jennings R, Baird C. Aortic uncrossing and tracheobronchopexy corrects tracheal compression and tracheobronchomalacia associated with circumflex aortic arch. J Thorac Cardiovasc Surg. 2020;160:796-804.

2. Planche $C$, Lacour-Gayet F. Aortic uncrossing for compressive circumflex aorta: 3 cases. Presse Med. 1984;13:1331-2.

3. Backer C, Monge M, Russell H, Popescu A, Rastatter J, Costello J. Reoperation after vascular ring repair. Semin Thorac Cardiovasc Surg Pediatr Card Surg Апnu. 2014;17:48-55.

4. Mitchell M. Anterior tracheal suspension. Oper Techn Thorac Cardiovasc Surg. 2011;16:331-9.

5. Mitchell M, Rumman N, Chun R, Rao A, Martin T, Beste D, et al. Anterior tracheal suspension for tracheobronchomalacia in infants and children. Ann Thorac Surg. 2014;98:1246-53. 